\title{
Biotech strikes back in Q3
}

\section{Walter Yang}

Financings for public biotechs returned to levels not seen in over two years. Excluding partnership funding, publicly traded companies raised $\$ 5.9$ billion in the third quarter. This is also the first quarter since the

\section{Stock market performance}

The BioCentury 100 and NASDAQ Biotechnology indexes were up 9\% and $12 \%$, respectively, whereas the Dow, NASDAQ and S\&P 500 were all up more than $15 \%$.

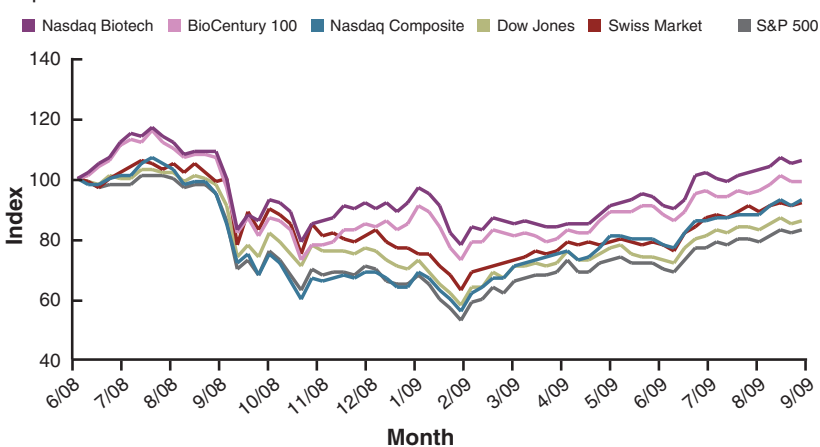

\section{Global biotech industry financing}

IPO and follow-on financings came back last quarter, with $\$ 657$ million from IPOs and $\$ 2.4$ billion from follow-ons. This compares to no IPOs and $\$ 851$ million in follow-ons in 3Q08.

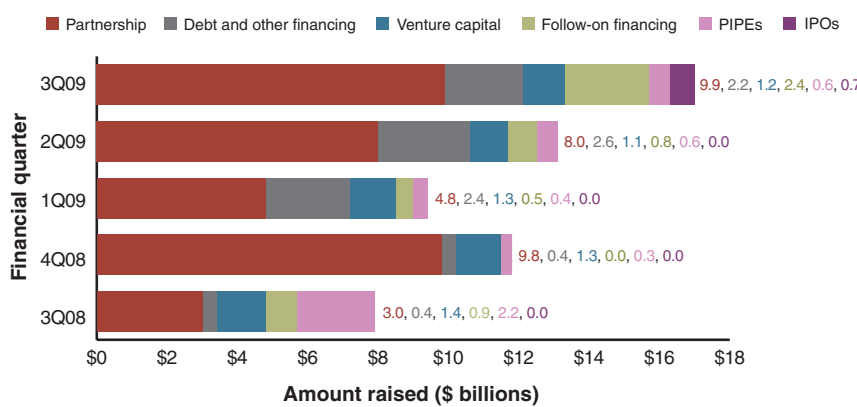

Partnership figures are for deals involving a US company. Source: BCIQ: BioCentury Online Intelligence, Burrill \& Co.

IPOs, initial public offerings; PIPEs, private investments in public equity.

Notable Q3 deals

\begin{tabular}{|c|c|c|c|c|c|c|c|c|c|}
\hline \multicolumn{5}{|c|}{ 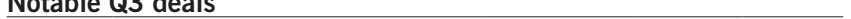 } & & & & & \\
\hline $\begin{array}{l}\text { Venture } \\
\text { capital }\end{array}$ & Company (lead investors) & $\begin{array}{l}\text { Amount raised } \\
\text { (\$ millions) }\end{array}$ & $\begin{array}{c}\text { Round } \\
\text { number }\end{array}$ & Date closed & $\begin{array}{l}\text { Mergers and } \\
\text { acquisitions }\end{array}$ & Target & Acquirer & Value ( $\$$ millions) & Date announced \\
\hline & Pacific Biosciences $^{1}$ (Deerfield, Intel) & $\$ 68.0$ & 5 & 12-Aug & & Sepracor & Dainippon Sumitomo & $\$ 2,600.0$ & 3-Sep \\
\hline & Calypso Medical (Skyline, Frazier) & $\$ 50.0$ & 5 & 17-Sep & & Medarex & Bristol-Myers Squibb & $\$ 2,400.0$ & 22-Jul \\
\hline & Enobia (OrbiMed) & $\$ 50.0$ & 3 & 10-Aug & & ESBATech & Alcon & $\$ 589.0$ & 14-Sep \\
\hline & Complete Genomics (Essex Woodlands, OrbiMed) & $\$ 45.0$ & 4 & 24-Aug & & Noven & Hisamitsu & $\$ 428.0$ & 14-Jul \\
\hline & Adamas (Aeris, DAG Ventures) & $\$ 40.0$ & 4 & 18-Aug & & Peplin & Leo Pharma & $\$ 287.5$ & 3-Sep \\
\hline & Zogenix (Clarus, Domain) & $\$ 36.0$ & 2 & 23-Sep & & DxS & Qiagen & $\$ 130.0$ & 22-Sep \\
\hline \multirow[t]{5}{*}{ IPOs } & \multicolumn{2}{|l|}{ Company (lead underwriters) } & \multicolumn{2}{|c|}{ Amount raised (\$ millions) } & \multicolumn{2}{|c|}{ Change in stock price since offer } & \multicolumn{3}{|l|}{ Date completed } \\
\hline & CanBas (Mitsubishi UFJ) & & \multicolumn{2}{|c|}{$\$ 14.8$} & \multicolumn{2}{|l|}{$61 \%$} & \multicolumn{3}{|l|}{ 17-Sep } \\
\hline & \multicolumn{2}{|l|}{ Cumberland (UBS, Jefferies, Wells Fargo) } & \multicolumn{2}{|c|}{$\$ 85.0$} & \multicolumn{2}{|l|}{$-5 \%$} & \multicolumn{3}{|l|}{ 10-Aug } \\
\hline & \multicolumn{2}{|l|}{ D-Pharm (Clal Finance) } & \multicolumn{2}{|c|}{$\$ 7.4$} & \multicolumn{2}{|l|}{$67 \%$} & \multicolumn{3}{|l|}{ 17-Aug } \\
\hline & \multicolumn{2}{|c|}{ Talecris $^{2}$ (Morgan Stanley, Goldman Sachs, Citigroup, JPMorgan) } & \multicolumn{2}{|c|}{$\$ 550.0$} & NA & & 30-Sep & & \\
\hline \multirow[t]{10}{*}{$\begin{array}{l}\text { Licensing / } \\
\text { collaboration }\end{array}$} & Researcher & Investor & $\begin{array}{c}\text { Value } \\
(\$ \text { millions })\end{array}$ & \multicolumn{6}{|l|}{ Deal description } \\
\hline & PTC Therapeutics & Roche & $\$ 1,924.0$ & \multicolumn{6}{|c|}{ Develop oral small molecules against four central nervous system disease (CNS) targets } \\
\hline & Nektar & AstraZeneca & $\$ 1,120.0$ & \multicolumn{6}{|c|}{ Worldwide rights to NKTR-118 for opioid-induced constipation and NKTR-119 for pain without causing constipation } \\
\hline & Algeta & Bayer & $\$ 800.0$ & \multicolumn{6}{|c|}{ Develop and commercialize Alpharadin radiopharmaceutical in phase 3 for bone metastases } \\
\hline & Acorda & Biogen Idec & $\$ 510.0$ & \multicolumn{6}{|c|}{ Ex-US rights to develop and commercialize multiple sclerosis compound Fampridine-SR } \\
\hline & Portola & Merck & $\$ 470.0$ & \multicolumn{6}{|c|}{ Worldwide rights to oral anticoagulant betrixabanin } \\
\hline & Crucell & Johnson \& Johnson & $>\$ 440.8$ & \multicolumn{6}{|c|}{ Develop and commercialize monoclonal antibodies and vaccines to treat and prevent influenza and other infectious and noninfectious diseases } \\
\hline & NeuroSearch & Johnson \& Johnson & $>\$ 346.2$ & \multicolumn{6}{|c|}{ Develop undisclosed therapeutics to treat various CNS diseases } \\
\hline & BioLineRx & Ikaria & $\$ 282.5$ & \multicolumn{6}{|c|}{ Worldwide rights to BL-1040 to prevent pathological cardiac remodeling after acute myocardial infarction } \\
\hline & Clinical Data & CombinatoRx & $\$ 252.0$ & \multicolumn{6}{|c|}{ Worldwide rights to ATL313 for use in a combination therapy to treat multiple myeloma and other B-cell cancers } \\
\hline
\end{tabular}

end of 2008 that money was raised through an initial public offering. Venture financing stayed level at about $\$ 1.2$ billion. Overall biotech indices underperformed compared with the Dow Jones and S\&P 500.

\section{Global biotech initial public offerings}

Last quarter marks the first time the industry raised money through IPOs since 4Q08.

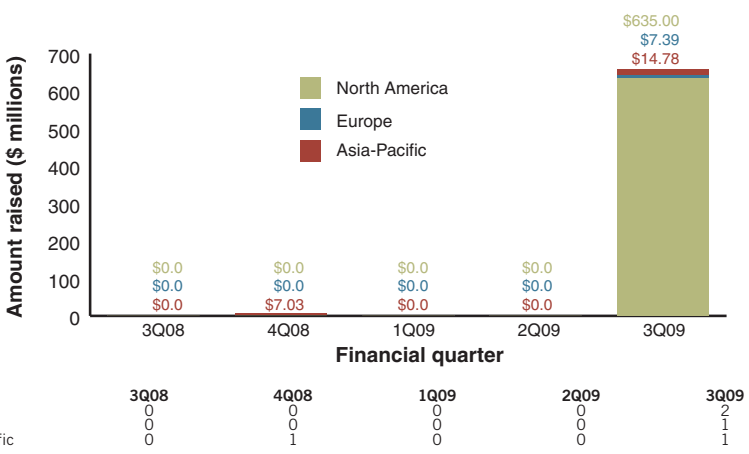

Table indicates number of IPOs. Source: BCIQ: BioCentury Online Intelligence

\section{Global biotech venture capital investment}

About $\$ 1.2$ billion was raised by private biotechs in $3 Q 09$, up $8 \%$ from 2Q09, but down $16 \%$ from a year ago.
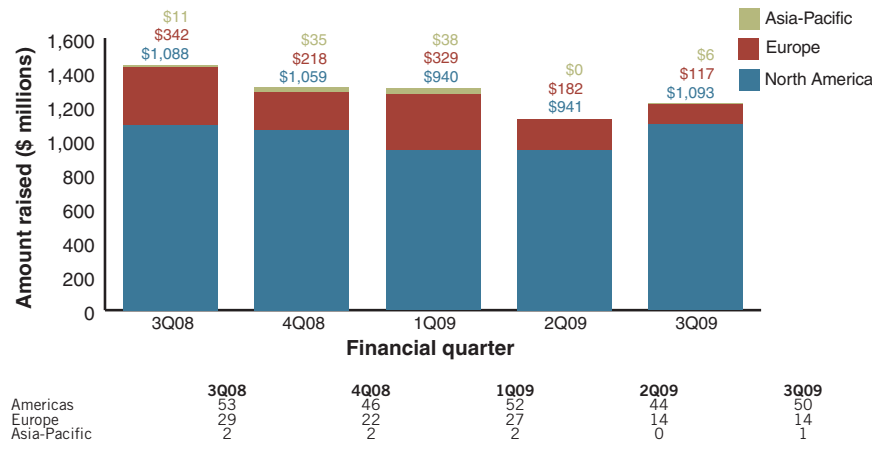

Table indicates number of $\mathrm{VC}$ investments and includes rounds where the amount raised was not disclosed. Source: $\mathrm{BCIQ}$ : BioCentury Online Intelligence

Walter Yang is research director at BioCentury 